\title{
Comparative Study of the Silk Fiber Structure and Properties before and after the Tensional Twisting Treatment *
}

\author{
Shun-Lin $\mathrm{Fu}^{*}$, Qiao-ming Dai, Jing Wang \\ College of Textile Science and Engineering, Wuhan Textile University, Wuhan 430200, China
}

\begin{abstract}
The production of the silk textile products with super softness and high suspension has become one of the hot and difficult topics in textile researches. To endow the silk fiber with improved softness, tensional drawing under different conditions was used to regulate the fiber structure and reduce the fiber initial modulus. The silk fiber structural and mechanical properties were tested before and after the tensional twisting treatments under wet and dry conditions respectively. The results showed that unlike the dry drafted twisting treatment, the pre-wet tensional twisting treatment induced more uniform helical structural formation with lower crystal destruction and molecule rupture than the dry one; though the breaking force decreased, the silk softness and breaking elongation were both improved after the pre-wet twisting treatment.
\end{abstract}

Keywords: Pre-wet Drawing; twisting; Silk Fiber; Structural Readjustment; Mechanical Properties

\section{Introduction}

In nature, spiders and several worms including mites, butterflies and moths, can produce silks. Among the glands of these organisms, there are some special epithelial cells that can synthesize synthetic fibrin, and these fibrins eventually become silk [1]. Silk fibers produced by silkworm Bombyx mori mainly composed of sericin and fibroin proteins and also contains a small amount of other amino acids and residues of various impurities: wax, fat, dyes and mineral salts [2-3]. According to the degumming treatment, the weight loss of the raw silk is treated as a sericin dissolved in the hot alkaline solution. The results showed that the fibroin content was ranged from $66.5 \%$ to $73.5 \%$ and the sericin content was $26.5 \%$ to $33.5 \%$ [4]. When Silkworms Bombyx mori produce their cocoon silk from two modified salivary glands, the sericin

${ }^{\star}$ Project supported by National Natural Science Foundation of China (No. 51403161) and the Technology Innovation Major Project of Hubei Province (No. 2166AAA024).

*Corresponding author.

Email address: 806654289@qq.com (Shun-Lin Fu). 
wraps the fibroin fibers with successive sticky layers that help to form the cocoon. Simultaneously, sericin glues silk threads together to ensure the cocoon cohesion. Sericin molecules with many hydrophilic groups are spherical. The small forces between sericin molecules encourage the silk sericin dissolving easily in water; the solubility increased dramatically as the water temperature increased. Therefore textile silk fibers can be obtained after the cocoon degumming in water [5-6]. Regarded as the queen of textiles, silk fibers are fine, long, elastic, renewable, biodegradable [7]. In addition to the conventional textile application, silk fibroin has been used to manufacture medical materials and food additives [8-10] due to its good biodegradability and biocompatibility [11], beauty products due to its unique physical properties, high resistance chemical properties [12]. Unlike the most advanced synthetic polymers, silk fiber production does not require harsh processing conditions [13]. Therefore, scientists from textile engineers, polymer chemists and biomedical researchers have paid great attention to silk. In recent years, silk fibroin has been widely used in the preparation of artificial skin, blood vessels, bone tissue, tendon [14]; it has also been applied to make the covering materials, controlled drug release carrier, tissue engineering scaffold and other biological materials [15-19].

Aforementioned silk appealing characteristics are closely related to the silk fiber internal structure and composition. Therefore, structural investigations were conducted on the silk before various extensive applications. Pe'rez-Rigueiroa. J. et al [20-21] submerged silkworm silk fibers in liquid environments (water, acetone, ethanol and isopropanol), and then carried out the tensile tests on them. Through a series of experiments, they made the conclusion that the water will destroy the hydrogen bond before the amorphous phase is present, while other solvents help to form new hydrogen bonds in the silk amorphous phase. They also performed mechanical tests on single brins of Bombyx mori silk and drew a conclusion that the sericin present in silk facilitated the fiber cross-sectional structure integrity with no contribution to the resistance of fiber tensile deformation. Chaoyang Jiang et al [22] investigated the mechanical properties of robust ultrathin silk fibroin films; the results indicted the methanol treatment could optimize the structure of thin silk film, achieving a high elastic modulus of 6.5 $\mathrm{GPa}$ and ultimate strength reaching $100 \mathrm{MPa}$ for the slik film. The structural optimization mechanism was regarded as the formation of a reinforced microstructure with crystalline bsheets serving as the reinforcing fillers and physical crosslink sites similar to bulk silk materials.

Under the pressure of the silk advantage features and wide biomedical applications, the silk should be employed to produce high qualified textile products with super softness and high suspension during the conventional textile application. To complete this task, the silk fiber softness required a primary improvement. We predicted pre-wet tensional twisting could reset the fiber internal molecular structure, reduce initial modulus and then improve flexibility. Thus the aim of the present study was to regulate the structure of molecular chains in the silk fiber and to endow helical conformation characteristics by using pre-wet tensional twisting. Then the silk fiber softness, stability and flexibility got improved. To accomplish this goal, the precious silk was selected to conduct the tensional twisting treatment under dry and wet conditions. The mechanical properties were tested for all the silk fibers before and after the corresponding treatments on a single fiber tensile strength tester. Moreover, the surface and inner structures were examined for all the silk fibers before and after the corresponding treatments by scanning electron microscopy and fourier transform infrared spectrometer. 


\section{Experimental}

\subsection{Materials}

Mulberry raw silk with about 20.0 tex fineness was provided by the Huguangshanse silk Co. Ltd., Jiangsu province, China. The silk samples with the length of $200 \mathrm{~mm}$ were glued on a black flannel board, and each black flannel board was affixed with twenty silk samples; nine black flannel boards were prepared totally. In this experiment, the water used was distilled water.

\subsection{Sample Preparation}

\subsubsection{Preparation of Silk Samples Using Dry Tensional Twisting}

For each sample, two clips were employed to clamp the silk two ends respectively; the length between the two clamp points remained the same as $200 \mathrm{~mm}$. Then one silk fiber end with a clip was fixed on the black flannel board by magnets (shown in Fig. 1(a)), the other fiber end with the other clip performed twisting to insert twists on the fiber sample by rotating the other clip. The silk samples were inserted with 0, 250, 500, 750 and 1000 twists/meter respectively. The twisted silk samples were smoothly put on a black flannel board until the twist distribution was steady. After the completion of the twisting process, silk samples $(200 \mathrm{~mm})$ were gently sprayed with distilled water until completely wet. Let the silk samples maintain wet state for five minutes at room temperature, then dry them with a blower. Thereafter a collection of every 20 silk samples were fixed on the holder with two magnets, and two clips were hanged in the center of the silk collection for 2 days (shown in Fig. 1(b)). This treatment was in order to give tension treatment (the applied tension was $2.55 \times 10^{-3} \mathrm{cN}$ ). Then the samples treated by dry tensional twisting were ready for tests (shown in Fig. 1(c)).

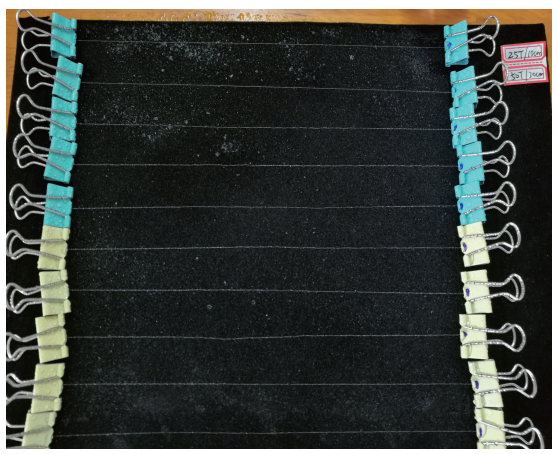

(a) Clamping fiber two ends with the same length

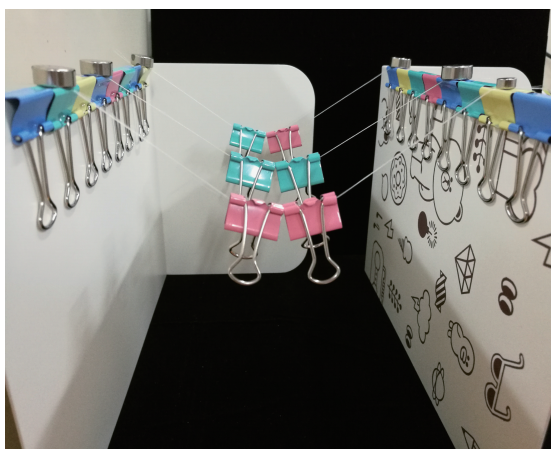

(b) Tensional treatment with clips after the twisting process

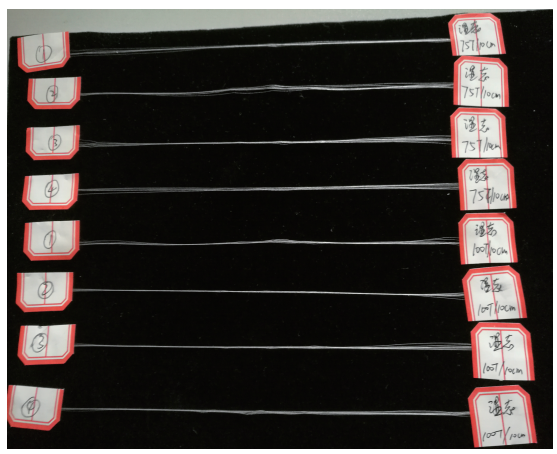

(c) Silk fiber samples after corresponding treatments

Fig. 1: Silk sample preparation process

\subsubsection{Preparation of Silk Samples Using Wet Tensional Twisting}

The silk samples were neatly put on the black flannel board and moisturize it completely with distilled water. The silk samples were soaked in water. Two clips were applied to clamp the end of the silk $(200 \mathrm{~mm})$, similar to the twisting procedure introduction in dry condition. The 
silk samples were finally twisted to $0,250,500,750$, and 1000 twists/meter respectively. Twist distribution remained in steady state. After the completion of the twisting process, drying and tensional treatment were also conducted using the same method with that for tensional twisting under dry condition (Fig. 1(b)). Then the samples treated by dry tensional twisting were ready for test.

\section{$2.3 \quad$ Measurement}

An Electronic strength tester for single fiber (FAVIMAT+BOBOT2 Type, Germany) was used to measure the mechanical properties of silk samples at $22{ }^{\circ} \mathrm{C}$ and $65 \% \mathrm{RH}$ with the clamping length of $20 \mathrm{~mm}$ and elongation rate of $20 \mathrm{~mm} / \mathrm{min}$. The surface structure of silk samples was examined on a scanning electron microscopy (SEM) instrument (S4800, Japan) at $15 \mathrm{kV}$ of acceleration voltage. Before placing the samples in the SEM chamber, the samples were mounted onto an aluminum stud and sputter-coated with gold/palladium for $180 \mathrm{~s}$ (E-1010 ION SPUTTER, Hitachi, Japan) to prevent charging. The spectra of samples were obtained by a FTIR instrument ((Nicolet 5700, Thermo electron Co., Ltd., US)) in the range of $6000-4500 \mathrm{~cm}^{-1}$. The samples were prepared in KBr pellets. A X-ray diffraction instrument (D8 ADVANCE X, Bruker, Germany) was employed to determine the crystalline structure of original sample and silk fibers after tensional twisting treatments. The scanning range was from 0 to 50 .

\section{Results and Discussion}

\subsection{Surface Structural Observation}

SEM was used to analyze the surface morphology of silk fibers before and after the dry and wet tensional twisting treatments. The longitudinal micro surface structures were obviously different between original and treated silk samples (shown in Fig. 2). It was observed that silk fibers arranged in parallel with each other and the sericin could be seen clearly at some junctions for the original samples. In addition, the original samples also had smooth and intact fiber surface features (Fig. 2(a)).

The longitudinal micro graphs showed the silk fibers got larger radical deformations as the twists increased (Fig. 2(b), 2(c), 2(d) and 2(e)). However, it could be observed that the twist of wet twisted silk was more uniform with less damage than the dry ones (Fig. 2(f), 2(g), 2(h) and 2(i)). This also indicated that the inner structure of wet twisted samples was relatively uniform, with same endurance to external force. In the contrary, the dry twisted silks showed fractional morphology which was a sign that the inner structure of the treated sample was not uniform, with low capability to endure external forces.

\subsection{Tensile Properties}

Fig. 3 showed the initial modulus of the silk fibers with different twists under different processing conditions. It was obvious the initial modulus of silk fibers decreased significantly after wet tensional twisting treatment; moreover, the initial modulus decreased as the twist ratio increased. For the silk fibers after dry tensional twisting treatment, their initial modulus slightly increased 

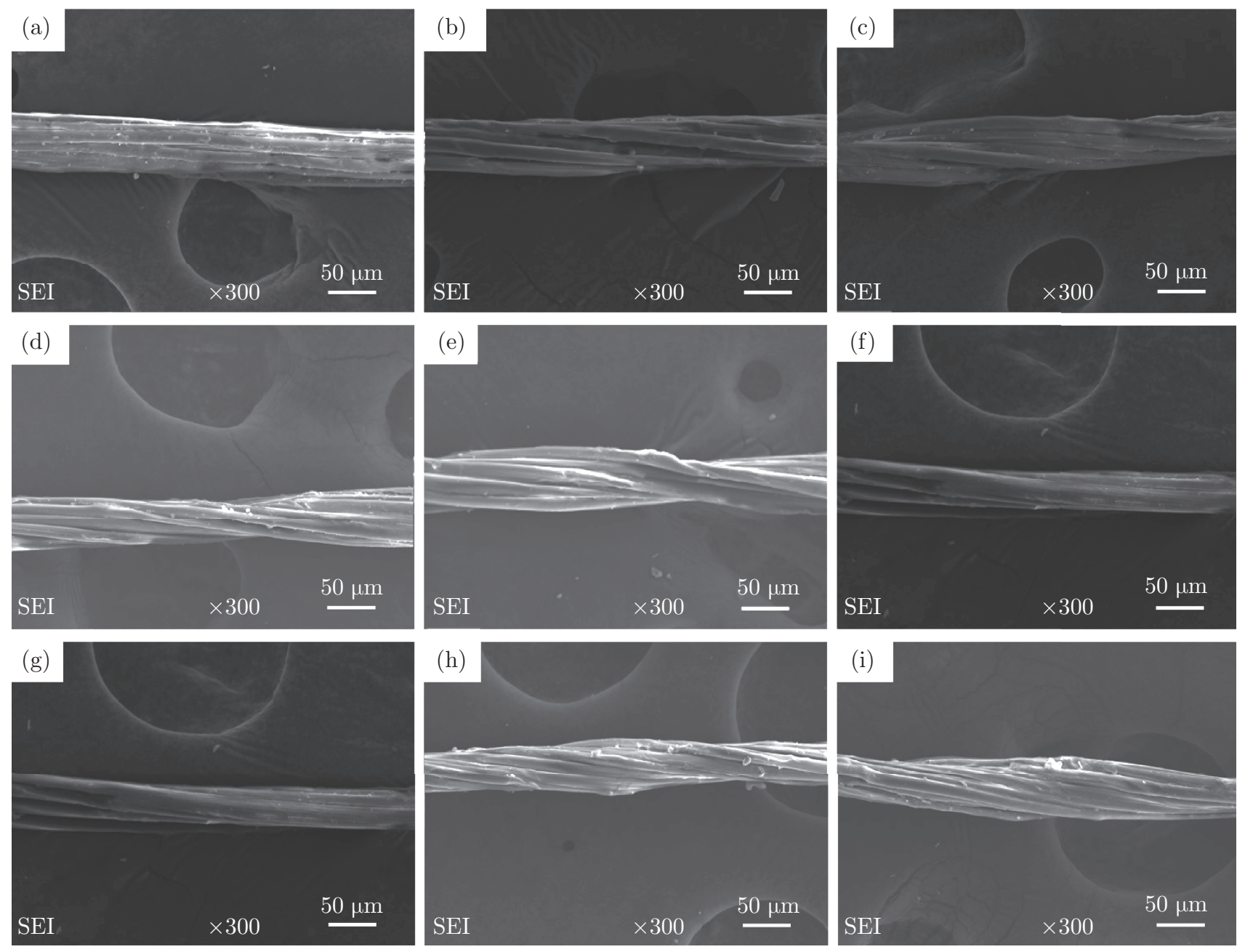

Fig. 2: SEM of (a) original silk fibres; (b) silk with 250 twists/meter dry tensional twisting; (c) silk with 500 twists/meter dry tensional twisting; (d) silk with 750 twists/meter dry tensional twisting; (e) silk with 1000 twists/meter dry tensional twisting; (f) silk with 250 twists/meter wet tensional twisting; (g) silk with 500 twists/meter wet tensional twisting; (h) silk with 750 twists/meter wet tensional twisting; (i) silk with 1000 twists/meter wet tensional twisting

at first and then decreased as twist ratio increased. In specialty, the initial modulus of silk fibers treated in dry condition was always much higher than the contrast in wet condition.

The reason for this phenomenon might be due to the common effect of distilled water and pre-tension process. When the silk fibers were immersed in distilled water for a period of time, the hydrogen bonds between the segments in the amorphous phase would suffer a certain degree of damage, leaving van der Waals bonds to dominate. Moreover, the silk fibers in water would promote the substitution of protein-protein hydrogen bonds by water-protein hydrogen bonds. The substitution of bonds between macromolecules should lead to the decrease in the initial modulus of the silk, consistent with other experimental observations [20]. During the pre-tensioning process, the breakage and recombination of the bonds might also occur between the molecular chains, leading to a reduction in the initial stiffness of silk fibers. For silk fibers twisted in dry condition, the destruction by external forces was gradually stronger than the own cohesion of itself with the twist degree increased. Accordingly, the distilled water could help produce the compliant fibers. Thus the fibers treated in the wet condition were softer than that treated in 


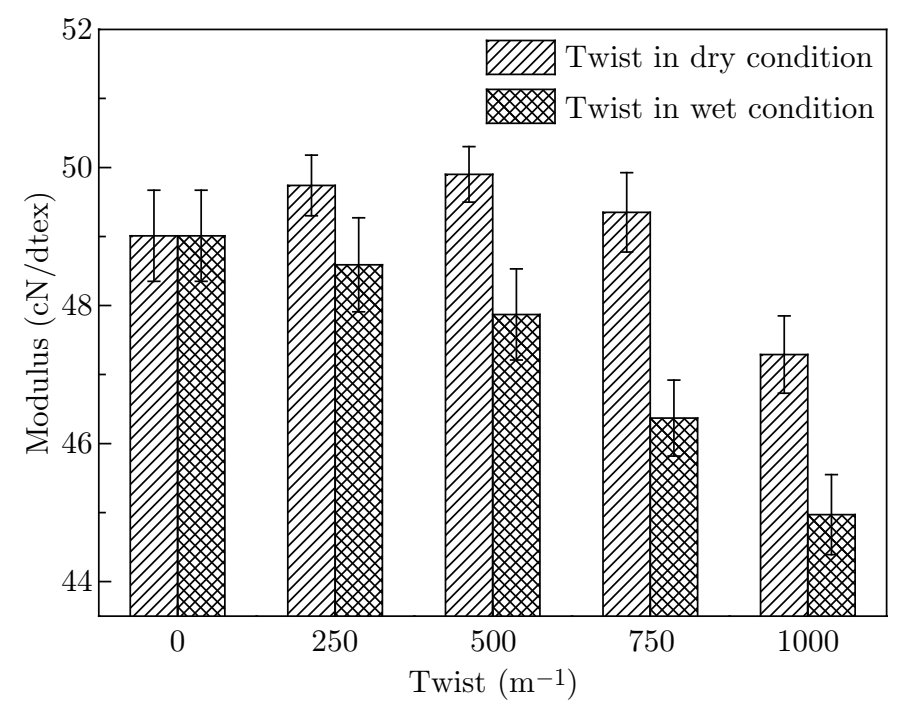

Fig. 3: The silk initial modulus comparison

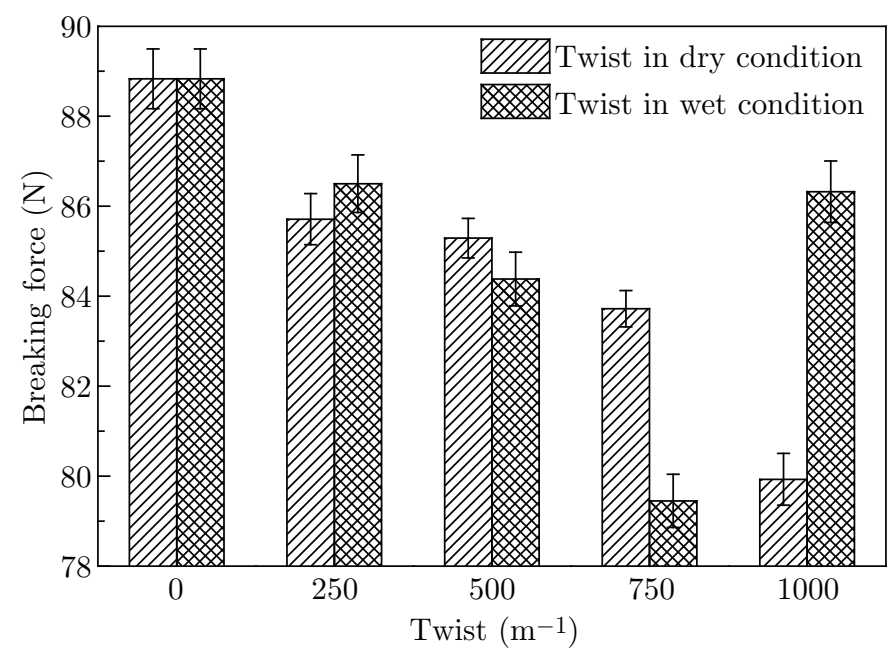

Fig. 4: The silk breaking force comparison

the dry condition.

The breaking forces were also compared for the fibers after dry and wet tensional twisting treatments (Fig. 4). No significant differences are apparent before silk fibers treated under the twisting level of 500 twists/meter. Above the level of 500 twists/meter, the breaking force of wet tensional twisting treated silk fibers reached a lowest point at 750 twists/meter and then rose sharply in the reverse direction; meanwhile the breaking force of dry tensional twisting treated silk fibers always kept in the declining trend.

The results of the breaking elongations were shown in Fig. 5 for the fibers after dry and wet tensional twisting treatments. Obviously the breaking elongation of wet tensional twisting treated silk fibers increased steadily with the inserted twist ratio increase. This could be attributed to the shrinkage of the twisted silk fibers without inner molecule damage in the wet condition. For dry tensional twisting treated silk fibers, the breaking elongation got a decline at first, which might be due to the increased compactness and damage of inter molecular bonds; thereafter the breaking elongation showed a rise, resulting from the increased fiber shrinkage of the twisting. 


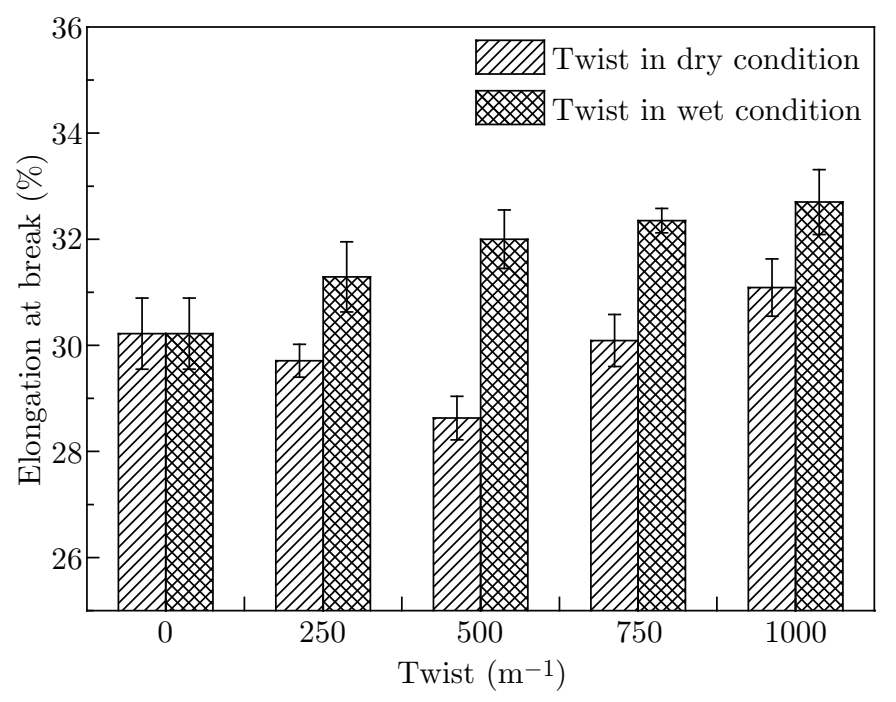

Fig. 5: The silk breaking elongation comparison

\subsection{Infrared Analysis}

Fourier transform infrared spectroscopy (FTIR) has been widely used to detect the molecular reaction and conformation transition of silk fibroin; the position and intensity of amide peaks can well indicate the molecular conformation [23-25]. The studies on the silk fibroin molecular conformation by Tsukada et al. [26-27] showed that silk fibroin was characterized by $\alpha$-helix absorption peaks around $1655 \mathrm{~cm}^{-1}$ (amide I), $1546 \mathrm{~cm}^{-1}$ (amide II), $1270 \mathrm{~cm}^{-1}$ (amide III), and $\beta$-sheet absorption around $1630 \mathrm{~cm}^{-1}$ (amide I), $1520 \mathrm{~cm}^{-1}$ (amide II) and $1240 \mathrm{~cm}^{-1}$ (amide III).

The FTIR spectrum of different silk fibers was shown in Fig. 6. The absorption peaks of three different silk fibers appear at $1654 \mathrm{~cm}^{-1}$ (amide I), $1508 \mathrm{~cm}^{-1}$ (amide II), $1227 \mathrm{~cm}^{-1}$ (amide III), $1161 \mathrm{~cm}^{-1}$ (amide III), $1066 \mathrm{~cm}^{-1}$ (amide IV). It was indicated that the main molecular conformations inside silk fiber through the twisting process are still $\beta$-helix structure. The infrared spectra characteristic peaks of silk before and after treatment remained unchanged. Therefore no new functional or chemical groups created after the wet and dry tensional twisting treatments. No difference occurred between the peaks. It seemed that the crystallinity degree of the fibers before and after treatment was not changed; or even if it changed, the change failed to be detected by FTIR. This indicated that the treatment did not have much influence on the conformation of $\beta$-sheet in the silk fibers. However, the characteristic peaks of (c) were more pronounced than (b), which was due to the fact that silk had excellent hydrophilic. In the process of wet treatment, under the influence of local stress, sufficient water and time, the chain segments of silk fibroin molecules could stretch repeatedly and rotate around the main axis. That encouraged the rearrangement of hydrogen bonds and the growth of $\beta$-sheet nuclei.

\subsection{X-ray Diffraction Analysis}

In order to elucidate the crystalline structure of aquatic silk fiber, WAXD profiles of untreated silk fiber and silk fiber after wet and dry tensional twisting treatments were recorded as shown in Fig. 7. The silk fiber before and after wet tensional twisting treated showed the similar X-ray 


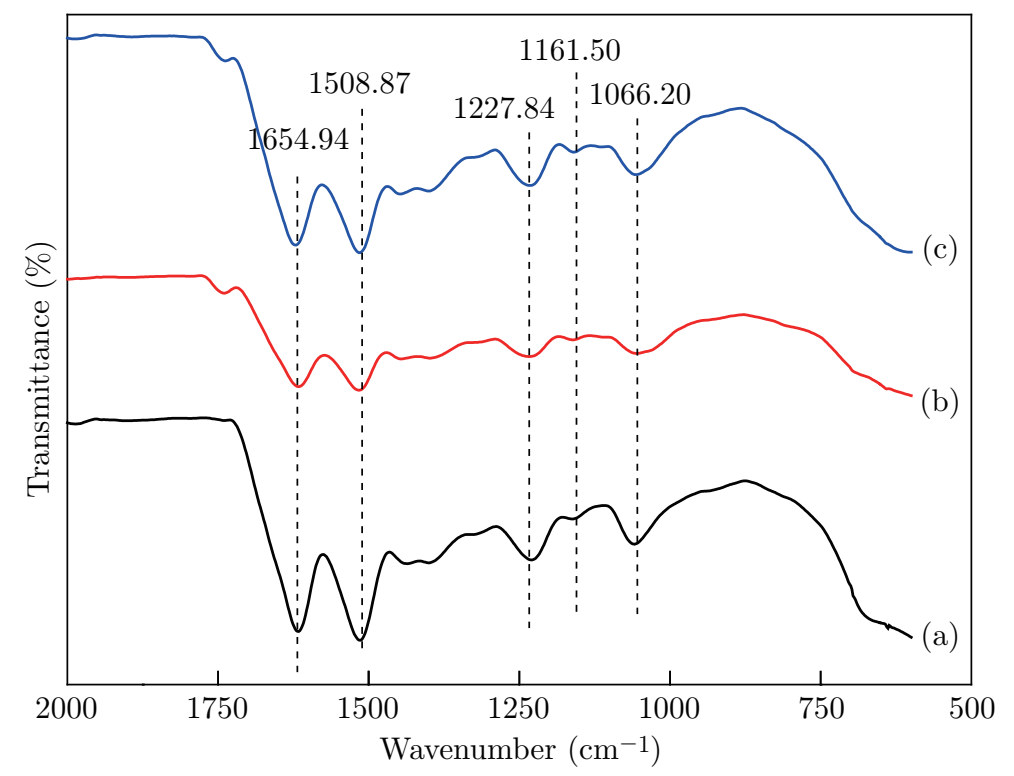

Fig. 6: FT-IR spectra of silk fibers before and after different treatments. (a) Untreated; (b) Wet tensional twisting treated; (c) Dry tensional twisting treated

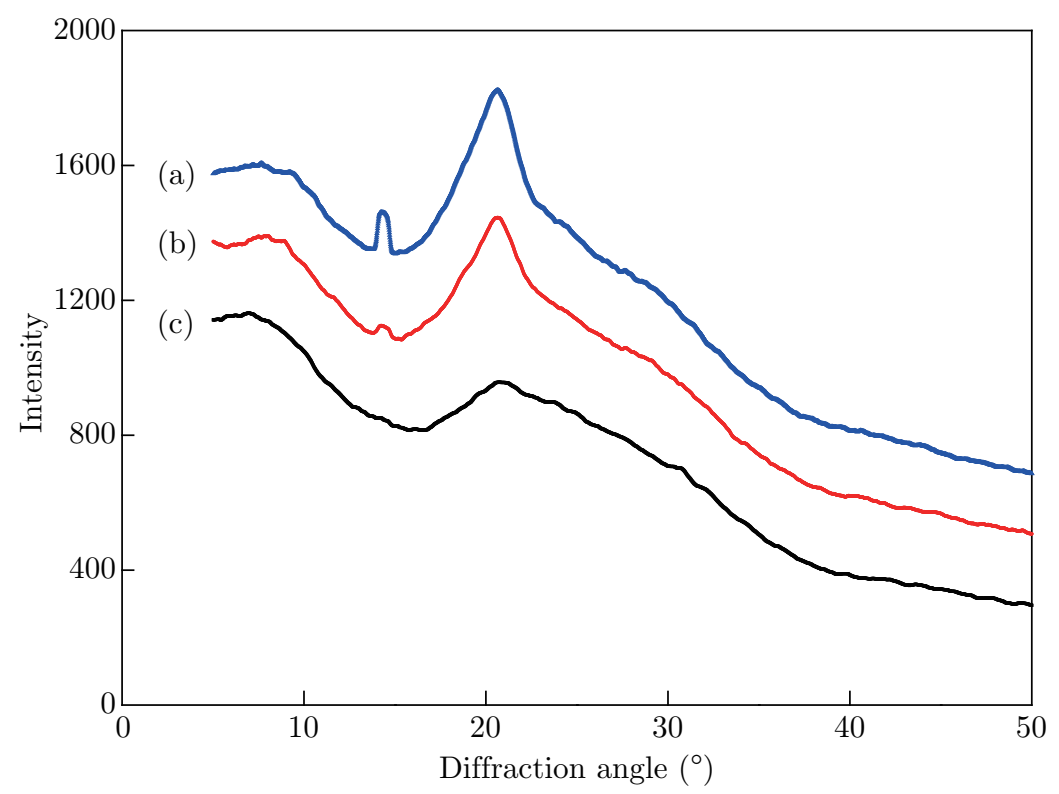

Fig. 7: X-ray diffraction curves of silk fibers. (a) Untreated; (b) Wet tensional twisting treated; (c) Dry tensional twisting treated

diffraction curves. The silk fiber before and after wet tensional twisting treated showed the similar X-ray diffraction curves. They both have major and eminent diffraction peaks at scattering angles $(2 \theta)$ of $20.8^{\circ}$. The crystalline structure of original silk fiber showed a striking resemblance to that after wet tensional twisting treated, suggesting similar crystalline structure for both samples. It was shown that the crystallinity of the wet tensional twisting treated fiber was slightly lower than original sample, which suggested that the effect of wet tensional twisting treatment caused damages for the silk samples was not obviously. While the crystallinity of the silk fiber treated with dry tensional twisting was significantly decreased. It indicated that the dry tensional twisting treatment could cause damages of the silk fiber inter molecular package structurest. 


\section{Conclusion}

According to the structure and performance characteristics of silk fibers, this paper purpose was to regulate the structure of molecular chains in the fiber and to endow helical conformation by tensional twisting treatments. The main conclusions achieved from the researches in this paper were summarized as follows:

(1) Tensional twisting treatments endowed the silk fibers with helical configuration structure; the wet treatment caused fewer fractures and induced more uniform helical distribution on silk fibers than the dry one.

(2) The initial modulus results validated the wet tensional twisting treatment induced a larger fiber softness improvement than the dry one. The silk breaking strength decreased as the softness increased after wet and dry tensional twisting treatments. In a certain range, the breaking strength of the dry-treated silk was higher than that of the wet-treated. But with the twist level increase, the wet-treated silk had a higher breaking strength than the dry-treated. The wet tensional twisting treatment always enhanced the original silk fiber breaking elongation; The dry tensional twisting treatment firstly reduced, then enhanced the original silk breaking elongation.

(3) FTIR results indicted no new functional or chemical groups were generated for the silk samples after wet and dry tensional twisting treatments.

(4) X-ray results showed that the dry tensional twisting treatment could cause damages of the silk fiber inter molecular package structures; while the wet tensional twisting treatment caused few damages for the silk samples.

\section{Acknowledgement}

We greatly acknowledge the support from the National Natural Science Foundation of China (Project No. 51403161) and the Technology Innovation Major Project of Hubei Province (Project No. 2166AAA024).

\section{References}

[1] Kaplan D, Mcgrath K, Kaplan D, et al. Protein-Based Materials. Q Rev Biol, 1998.

[2] Zhang Y Q. Applications of natural silk protein sericin in biomaterials. Biotechnol Adv, 2002, 20(2): 91-100.

[3] Sashina E S, Bochek A M, Novoselov N P, et al. Structure and solubility of natural silk fibroin. Russ J Appl, 2006, 79(6): 869-876.

[4] Cheung H Y, Lau K T, Ho M P, et al. Study on the Mechanical Properties of Different Silkworm Silk Fibers. Journal of Composite Materials, 2009, 43(43): 2521-2531. Iizuka, E. J Appl Polym Sci 1985, 41, 173.

[5] Magoshi, J.; Nakamura, S. J Appl Polym Sci 1985, 1, 187.

[6] Reddy RM. Innovative and Multidirectional Applications of Natural Fibre, Silk - A Review. Acad J Entomol, 2009.

[7] Hoppen HJ, Leenslag JW, Pennings AJ, et al. Two-ply biodegradable nerve guide: basic aspects of design, construction and biological performance. Biomaterials, 1990, 11(4): 286-90. 
[8] Moy RL, Lee A, Zalka A. Commonly used suture materials in skin surgery. Am Fam Physician, 1991, 44(6): 2123-2128.

[9] Tamapa A, et al. Application of the Bacterial Milk-Clotting Enzyme Preparation "Sirenin" in the Production of Soft and Solid Cheese Prepared with Sheep and Cow Milk. Int J Bio Macromol, 1987, 2(4): 35-40.

[10] Chen K, Iura K, Takano R, et al. Effect of fibroin administration on the blood cholesterol level of rats loaded with cholesterol. Journal of Insect Biotechnology \& Sericology, 2016, 62.

[11] Raghu K, Raghu K, Noorunnisa Khanam P, Venkata Naidu S. Chemical Resistance Studies of Silk/Sisal Fiber-Reinforced Unsaturated Polyester-Based Hybrid Composites. J Reinf Plast Comp, 2010, 29(3): 343-345.

[12] Pérez-Rigueiro J, Viney C, Llorca J, et al. Mechanical properties of silkworm silk in liquid media. Polymer, 2000, 41(23): 8433-8439.

[13] Asakura T, Suzuki Y, Nakazawa Y, et al. Silk structure studied with nuclear magnetic resonance [J]. Prog Nucl Mag Res Sp, 2013, 69(2): 23.

[14] Chiarini A, Petrini P, Bozzini S, et al. Silk fibroin/poly(carbonate)-urethane as a substrate for cell growth: in vitro interactions with human cells. Biomaterials, 2003, 24(5): 789.

[15] Armato U, Migliaresi C, Motta A. Bio-artificial substrate for the production of animal and, in particular, human tissse and organs: wo, ep1218490. 2002.

[16] Sofia S, Mccarthy MB, Gronowicz G, et al. Functionalized silk-based biomaterials for bone formation. J BIOMED MATER RES B, 2001, 54(1): 139.

[17] Gomes S, Leonor I B, Mano J F, et al. Silk-Based Biomaterials. Biomimetic Approaches for Biomaterials Development, 2012: 75-92.

[18] Li M, Ogiso M, Minoura N. Enzymatic degradation behavior of porous silk fibroin sheets. Biomaterials, 2003, 24(2): 357-365.

[19] Chen Z, Kimura M, Suzuki M, et al. Synthesis and Characterization of New Acrylic Polymer Containing Silk Protein. FIBER, 2003, 59(5): 168-172.

[20] Pérez-Rigueiro J, Viney C, Llorca J, et al. Mechanical properties of single-brin silkworm silk. J APPL POLYM SCI, 2000, 75(10): 1270-1277.

[21] Magoshi J, Magoshi Y, Nakamura S, et al. Physical properties and structure of silk. V. Thermal behavior of silk fibroin in the random-coil conformation. J Polym Sci Pol Phys, 1977, 15(9): 16751683.

[22] Jiang C, Wang X, Gunawidjaja R, et al. Mechanical Properties of Robust Ultrathin Silk Fibroin Films. Adv Funct Mater, 2007, 17(13): 2229-2237.

[23] Odian G, O'Callaghan M P, Chien C K, et al. Zwitterion polymerization of tetrahydro-1-[4hydroxy-3-(2-hydroxyethoxy)phenyl] thiophenium hydroxide inner salt. Macromolecule, 1990, 23(4): 918-925.

[24] Gotoh Y, Tsukada M, Minoura N. Chemical modification of silk fibroin with cyanuric chlorideactivated poly (ethylene glycol): analyses of reaction site by 1H-NMR spectroscopy and conformation of the conjugates. BIOCONJUGATE CHEM, 1993, 4(6): 554.

[25] Li M, Tao W, Lu S, et al. Compliant film of regenerated Antheraea pernyi silk fibroin by chemical crosslinking. Int J Biol Macromol, 2003, 32(3-5): 159-163.

[26] Tao W, Li M, Zhao C. Structure and properties of regenerated Antheraea pernyi silk fibroin in aqueous solution. Int J Biol Macromol, 2007, 40(5): 472.

[27] Huang D, Peng Z, Hu Z, et al. A new consolidation system for aged silk fabrics: Effect of reactive epoxide-ethylene glycol diglycidyl ether. React Funct Polym, 2013, 73(1): 168-174. 